IOSR Journal of Pharmacy

e-ISSN: 2250-3013, p-ISSN: 2319-4219, www.iosrphr.org

Volume 2 Issue 6 || $\mid$ Nov-Dec. 2012 || $\mid$ PP.61-70

\title{
Neuroprotective effect of Zingiber officinale in 3-np-induced huntington disease
}

\author{
Manisha Sharma, Nidhi Sharma, Ramica Sharma \\ Rayat institute of Pharmacy, Dept. of Pharmacology Punjab (India). (AP)
}

\begin{abstract}
Huntington's disease (HD) is an autosomal dominant inherited neurodegenerative disease that causes clinical manifestations such as progressive choreiformic movements, cognitive abnormality and psychiatric deterioration. HD results from the destruction of neurons in the basal ganglia, and oxidative stress, Exitotoxicity and energy impairment hasbeen implicated in its pathogenesis. 3-Nitropropionic acid (3NP), a potent neurotoxin, has been reported to induce oxidative/ nitrosative stress and causes neurobehavioral and biochemical changes that mimic HD in humans. In the present work, we evaluated the effects of a well-known antioxidant on behavioral, biochemical, and mitochondrial dysfunction induced by 3NP. The study was designed to investigate the neuroprotective effects of Ethanolic extract of Zingiber oficinale against 3-NP-induced neurotoxicity. Intraperitoneal administration of 3-NP (20 mg/kg for 7 days) caused a decline in motor function (locomotor activity and impaired rotarod activity). Chronic treatment with EEZO root extracts (100 and $200 \mathrm{mg} / \mathrm{kg}$ ) for 1 week dose-dependently improved 3-NP-induced behavioral, biochemical, and enzymatic changes $(P<.05)$. Biochemical analysis revealed that systemic 3-NP administration significantly increased lipid peroxidation and nitrite and acetylcholiestrease level, Chronic administration of EEZO (100 and $200 \mathrm{mg} / \mathrm{kg}$ ) dose-dependently restored biochemical alterations induced by chronic 3-NP treatment (P<.05). These findings suggest that neuroprotective actions of Zingiber officinale are mediated via its anticholinesterase activity. However, further studies are required to elucidate the molecular mechanisms involved in order to support the clinical use of the plant extract as a therapeutic agent for the treatment of $\mathrm{HD}$.
\end{abstract}

Keywords-Huntington's disease, oxidative stress, Zingiber officinale, Neuroprotective, 3-nitropropionic acid.

\section{INTRODUCTION}

Huntington's disease (HD) is an autosomal dominantly inherited progressive neurodegenerative disorder, characterized by progressively worsening chorea, psychiatric disturbances, cognitive impairment and weight loss $(1,2)$. First reported by George Huntington in 1872 the degenerative process primarily involves medium spiny striatal neurons (MSN) and to a lesser extent cortical neurons (3).Cognitive deficits are core neuropsychiatric problem among HD patients (4,5). HD patients often exhibit deficits in executive tasks requiring planning, problem solving, and cognitive flexibility with progress of the disease $(6,7)$. HD involve the generation of excitotoxic events, the alteration in energy metabolism and mitochondrial dysfunction, the resulting oxidative stress, and the htt aggregation, all leading to neurotoxicity(8). 3-Nitropropionic acid (3-NP) is a well known fungal toxin causing neurotoxicity in both animals and humans (9,10).3-NP systemic administration has been proposed to produce 3-NP treatment induces memory impairment in rodents as one of the symptoms of HD further related to an increase in oxidative stress specially glutathione levels (11). Although, the precise mechanism of neuronal degeneration is not yet perfectly understood however, experimental evidences demonstrate that 3-NP administration produce lesions in striatum and cortex areas of the brain.(12,13). Besides, oxidative damage has been reported to play an important role in 3-NP induced neurotoxicity. 3-NP depletes antioxidant defense enzymes and increases levels of reactive oxygen/nitrogen species in different areas of the brain $(14,15)$ These observations have been further strengthened by successful use of several endogenous and exogenous antioxidants against 3-NP induced neuronal damage in rats .It has been further reported that oxidative damage occurs at early stage of 3-NP toxicity in addition to mitochondrial dysfunction $(16,17,18)$. Zingiber officinale is widely used around the world in foods as a spice. For centuries, it has been an important ingredient in Chinese, Ayurvedic and Tibbi-Unaniherbal medicines for the treatment of catarrh, rheumatism, nervous diseases, gingivitis, toothache, asthma, stroke, constipation an diabetes, hypertension , antiplatelet ,nausea ,antiemetic , antihyperlipidemic (19-21). Several reviews have appeared in the literature about this plant, and this may reflect the popularity of the subject and its common use as a spice 
and a medicinal plant $(22,23)$. It has been demonstrated that the plant posses a potent antioxidant activity (24).But no evidences are still present that indicate its importance in the management of HD.Hence the study has been designed to explore the neuroprotective effect of Zingiber officinale against 3-NP-induced Huntington's disease.

\subsection{Animals}

\section{MATERIALS AND METHODS}

The experimental protocol used in the present study was approved by the Institutional Animal Ethical Committee. Age matched young Wistar rats weighing about 150-200 g were employed in the present study. They were fed on standard chow diet and water ad libitum. They were acclimatized in the institutional animal house and were exposed to normal cycle of day and night.

\subsection{Drugs and treatment schedule}

- 3-Nitropropionic acid (3- NP) ((Sigma USA) Ethanolic extract of Zingiber officinal solutions were made freshly at the beginning of each experiment. A solution of $20 \mathrm{mg} / \mathrm{kg} \mathrm{3-} \mathrm{NP}$ was prepared in normal saline and administered in a volume of $1 \mathrm{ml} / 100 \mathrm{~g}$ body weight intraperitoneally (i.p.). EEZO was suspended in $0.5 \%$ sodium carboxymethylcellulose (CMC)and administered $1 \mathrm{ml} / 100$ gbody weightperorally(p.o.). Animals were randomly divided into five groups of 6 each: Group 1 (Normal group) received vehicle for EEZO (perorlly) and also normal saline (intraperitoneally); Group 2 received 3-NP $(20 \mathrm{mg} / \mathrm{kg}$, i.p.) for 7 days; Group 3 received EEZO $(100 \mathrm{mg} / \mathrm{kg})+3-\mathrm{NP}(20 \mathrm{mg} / \mathrm{kg}$, i.p. $)$ for 7 days; Group 4 received EEZO $(200 \mathrm{mg} / \mathrm{kg})+3-\mathrm{NP}(20 \mathrm{mg} / \mathrm{kg}$, i.p.) for 7 days; and Group 5 received EEZO (200 mg/kg) alone for 7 days. In Groups 3 and 4, EEZO was given 1 hour prior to 3-NP administration, and the doses were selected on the basis of previous studies (24).

\subsection{Behavior assessment}

\subsubsection{Morris water maze}

The acquisition and retention of memory was examined using Morris Water Maze test(25)

\subsubsection{Assessment of locomotor activity}

The locomotor activity was monitored using Digital Actophotometer apparatus. Each interruption of a beam on the $\mathrm{x}$ - or $\mathrm{y}$-axis generated an electric impulse, which was presented on a digital counter. The apparatus was placed in a darkened, light and sound attenuated and ventilated testing room. Each animal was observed over a period of $5 \mathrm{~min}$ and values expressed as counts per $5 \mathrm{~min}$ (26).

\subsubsection{Rota Rod Activity}

All animals were evaluated for grip strength by using the rotarod. Each rat was given a prior training session before initialization of therapy to acclimatize them on a rotarod apparatus. Animal was placed on the rotating rod with a diameter of $7 \mathrm{~cm}$ (speed $25 \mathrm{rpm}$ ). Three separate trials were given to each rat at $5 \mathrm{~min}$ interval and cut off time (180) was maintained throughout the experiment. The average results were recorded as fall of time (27).

\subsubsection{Elevated plus maze}

Another paradigm to assess memory dysfunction is elevated plus maze (28).

\subsection{Biochemical study}

\subsubsection{Preparation of homogenate}

For the biochemical analysis, animals were scarified by decapitation immediately after behavioural assessment. The brains were removed. $10 \%(\mathrm{w} / \mathrm{v})$ tissue homogenate was prepared in $0.1 \mathrm{M}$ phosphate buffer (pH 7.4). The homogenate was centrifuged at $10,000 \mathrm{~g}$ for $15 \mathrm{~min}$ and clear supernatant was separated and used for biochemical estimations (29).

\subsubsection{Measurement of lipid peroxidation}

Malondialdehyde (MDA) which is a measure of lipid peroxidation was measured as described by Ohkawa. Briefly, brain tissues were homogenized with 10 times (w/v) 0.1 sodium phosphate buffer ( $\mathrm{pH} 7.4$ ). The reagents acetic acid $1.5 \mathrm{ml}(20 \%) \mathrm{pH} 3.5,1.5 \mathrm{ml}$ thiobarbituric acid $(0.8 \%)$ and $0.2 \mathrm{ml}$ sodium dodecyl sulfate $(8.1 \%)$ were added to $0.1 \mathrm{ml}$ of processed tissue sample. The mixture was then heated at $1000 \mathrm{C}$ for 60 mins. The mixture was cooled with tap water and $5 \mathrm{ml}$ of n-butanol: pyridine $(15: 1 \% \mathrm{v} / \mathrm{v})$, and $1 \mathrm{ml}$ of distilled water were added. The mixture was shaken vigorously. After centrifugation at $4000 \mathrm{rpm}$ for $10 \mathrm{mins}$, the organic layer was withdrawn and absorbance was measured at $532 \mathrm{~nm}$ (30).

\subsubsection{Estimation of acetylcholinesterase (AChE) levels}

The quantitative measurement of acetylcholinesterase levels in brain was performed according to the method of Ellman et al. The assay mixture contained $0.05 \mathrm{ml}$ of supernatant, $3 \mathrm{ml}$ of $0.01 \mathrm{M}$ sodium phosphate buffer (pH 8), $0.10 \mathrm{ml}$ of acetylthiocholine iodide and $0.10 \mathrm{ml}$ of 5,5'dithio-bis(2-nitrobenzoic acid(DTNB). The change in absorbance was measured immediately at $412 \mathrm{~nm}$ using spectrophotometer. Protein estimation was done by biuret method(31). 


\subsubsection{Estimation of nitrite}

The accumulation of nitrite in the supernatant, an indicator of the production of nitric oxide (NO) was determined using a colorimetric assay with the Griess reagent $(0.1 \% \mathrm{~N}-(1$-naphthyl) ethylenediamine dihydrochloride, $1 \%$ sulfanilamide and $2.5 \%$ phosphoric acid) as described by Green et al. Equal volumes of supernatant and the Griess reagent were mixed. Then, the mixture was incubated for $10 \mathrm{~min}$ at room temperature in the dark, and the absorbance was measured at $540 \mathrm{~nm}$ using a spectrophotometer (32).

\subsubsection{Estimation of reduced glutathione (GSH)}

Reduced glutathione in the brain was estimated according to the method described by Ellman. One milliliter of supernatant was precipitated with $1 \mathrm{ml}$ of $4 \%$ sulfosalicylic acid and cold digested at $4^{\circ} \mathrm{C}$ for $1 \mathrm{~h}$. The samples were centrifuged at $1,200 \times \mathrm{g}$ for $15 \mathrm{~min}$ at $4^{\circ} \mathrm{C}$. One milliliter of this supernatant was added to 2.7 $\mathrm{ml}$ of phosphate buffer $(0.1 \mathrm{M}, \mathrm{pH} 8)$ and $0.2 \mathrm{ml}$ of DTNB. A yellow color developed that was read immediately at $412 \mathrm{~nm}$ using a Perkin Elmer Lambda 20 spectrophotometer. Results were calculated using the molar extinction coefficient of chromophore $(1.36 \times 104 \mathrm{M}-1 \mathrm{~cm}-1)$ and expressed as a percentage of control (33).

\subsubsection{Protein estimation}

The protein content was measured by the biuret method using protein kit (34).

\subsection{Effect of EEZO on locomotor activity}

\section{RESULTS}

3-NP treatment caused a significant decrease in locomotor activity as compared to vehicle treated group (P<0.05). Further, chronic treatment with Z.O (100 and $200 \mathrm{mg} / \mathrm{kg}$, p.o.) significantly improved locomotor activity in 3-NP treated rats (Fig. 1). However lower dose of EEZO (100 mg/kg) did not show any significant effect on locomotor activity as compared to 3-NP treated rats. Z.O (200 mg/kg, p.o.) per se treatment did not show any significant change as compared to vehicle treated rats (Fig 1).

\subsection{Effect of EEZO on rotarod activity in 3-NP-treated rats}

$3-\mathrm{NP}(20 \mathrm{mg} / \mathrm{kg})$ treatment decreased muscle grip strength as assessed by the rotarod test on day $1^{\text {st }}$ $, 3^{\text {rd }}, 5^{\text {th }}, 7$ th day compared to the vehical group, which did not show any change in the activity of the animal throughout the experiment. EEZO $(200 \mathrm{mg} / \mathrm{kg}$, p.o.) alone did not alter grip strength of the animals compared to control animals $(\mathrm{P}<.05)$. Further, EEZO $(100$ and $200 \mathrm{mg} / \mathrm{kg}$, p.o.) treatment significantly improved muscle grip strength of 3-NP $(20 \mathrm{mg} / \mathrm{kg})$-treated rats $(\mathrm{P}<.05)$ (Fig 2$)$.

\subsection{Effect of EEZO on memory performance in elevated plus maze paradigm}

In elevated plus task the 3-NP treatment significantly delayed retention transfer latency as compared to vehicle treated group. In contrast, chronic administration of EEZO $(100,200 \mathrm{mg} / \mathrm{kg}$, p.o.) significantly shortened mean retention latencies in 3-NP treated groups (Fig. 3). However, EEZO $(200 \mathrm{mg} / \mathrm{kg}$, p. o.) per se treatment did not show any significance on transfer latency as compared to vehicle treated group.

\subsection{Effect of EEZO on spatial navigation task in 3-NP treated rats}

In Morris water maze test, the mean escape latency of trained rats gradually decreased during training session from day 1st to 3rd in 3-NP treated animals. However, escape latency was significantly increased on the $5^{\text {th }}$ and 7th day in Morris water maze as compare do the vehicle treated group $(\mathrm{P}<0.05)$. Chronic EEZO (200 $\mathrm{mg} / \mathrm{kg}$, p. o.) treatment showed a significant improvement in memory performance on the 5 th and 7 th day as compared to the 3-NP treated group $(\mathrm{P}<0.05)$ (Fig 4).

\section{BIOCHEMICAL ASSESSMENTS}

\subsection{Effect of EEZO on acetyl cholinesterase levels in 3-NP treated rats}

Chronic 3-NP treatment significantly increased acetylcholinesterase enzyme level in rat brain as compared to the normal control (P < 0.05). EEZO (100 and $200 \mathrm{mg} / \mathrm{kg}$, p.o.) treatment significantly attenuated acetylcholinesterase enzyme activity as compared to 3-NP treated rat (Fig. 5).EEZO(100 and $200 \mathrm{mg} / \mathrm{k}$.) per se treatment did not produce any significant effect on acetylcholinesterase enzyme activity as compared to 3-NP treated rats.

\subsection{Effect of EEZO on brain lipid peroxidation level}

Administration of 3-NP showed significant increase in brain MDA level in disease groups compared to normal control $(\mathrm{P}<0.001)$. Pretreatment with EEZO. at a dose of 100 and $200 \mathrm{mg} / \mathrm{kg}$ respectively showed significant decrease in the brain LPO level as compared with disease group $(\mathrm{P}<0.05)$ It shows that $200 \mathrm{mg} / \mathrm{kg}$ dose produce maximum significant as compared to $200 \mathrm{mg} / \mathrm{kg}$. (Fig. 6)

\subsection{Effect on brain reduced glutathione (GSH)}

GSH level in the brain homogenate of 3-NP administered rats showed significant decrease as compared with Normal Group $(\mathrm{P}<0.01)$. In 3-NP induced Huntington's disease in rats, pre treatment with the ethanolic extract of Z.O(.EEZO)(100 and $200 \mathrm{mg} / \mathrm{kg})$ showed significant increase in brain GSH level as compared to 
disease group $(\mathrm{P}<0.01)$. It shows that $200 \mathrm{mg} / \mathrm{kg}$ dose produce maximum significant as compared to $100 \mathrm{mg} / \mathrm{kg}$ (Fig. 7).

4.4. Effect of EEZO on brain nitrite levels in 3- nitropropionic acid-treated rats

Systemic 3-NP administration caused a significant increase in brain nitrite levels, which was significantly reversed by pretreatment with ZO $(100 \mathrm{mg} / \mathrm{kg}, 200 \mathrm{mg} / \mathrm{kg}$ orally $)$ and $200 \mathrm{mg} / \mathrm{kg}$ shows maximum effect .No significant effect of EEZO (200mg/kg, orally) alone was observed on brain nitrite levels as compared with normal (Fig 8)

4.5. Histological examination

A significant histopathological changes were seen in the brain of rats administered 3-NP as compared to normal rats. However, rats treated with EEZO $(100 \mathrm{mg} / \mathrm{kg}$ and $200 \mathrm{mg} / \mathrm{kg})$ significantly protected brain of rat indicated by reduction in inflammation, necrosis and glosis (Fig 9)
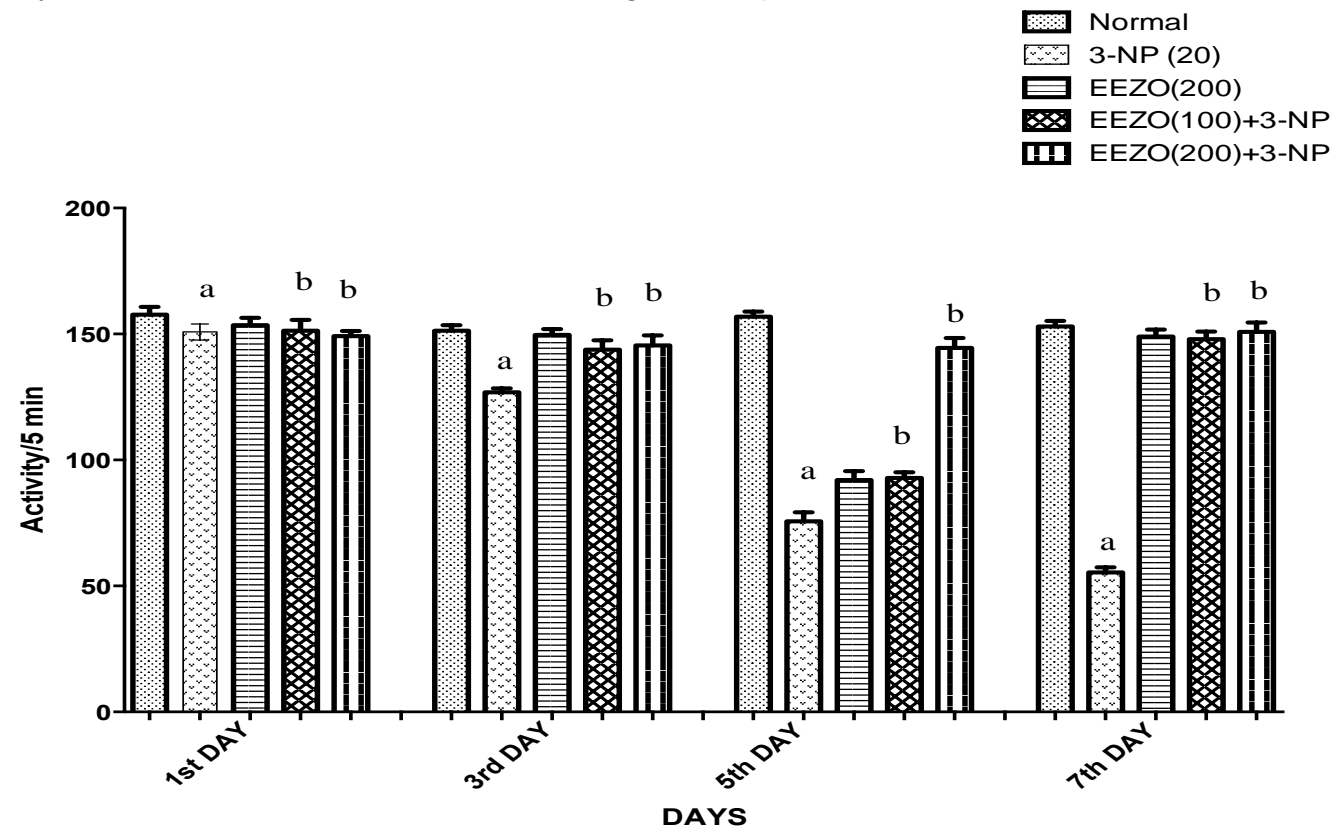

Fig 1Effect of EEZO $(100$ and $200 \mathrm{mg} / \mathrm{kg})$ on locomotor activity in 3-NP administered rats. Data are mean SEM values ( $n=6$ in each group). $a=p<0.05$ vs. compared to vehicle-treated normal group; $b=p<0.05$ compared to 3-NP-injected group (one-way ANOVA followed byTukey test)

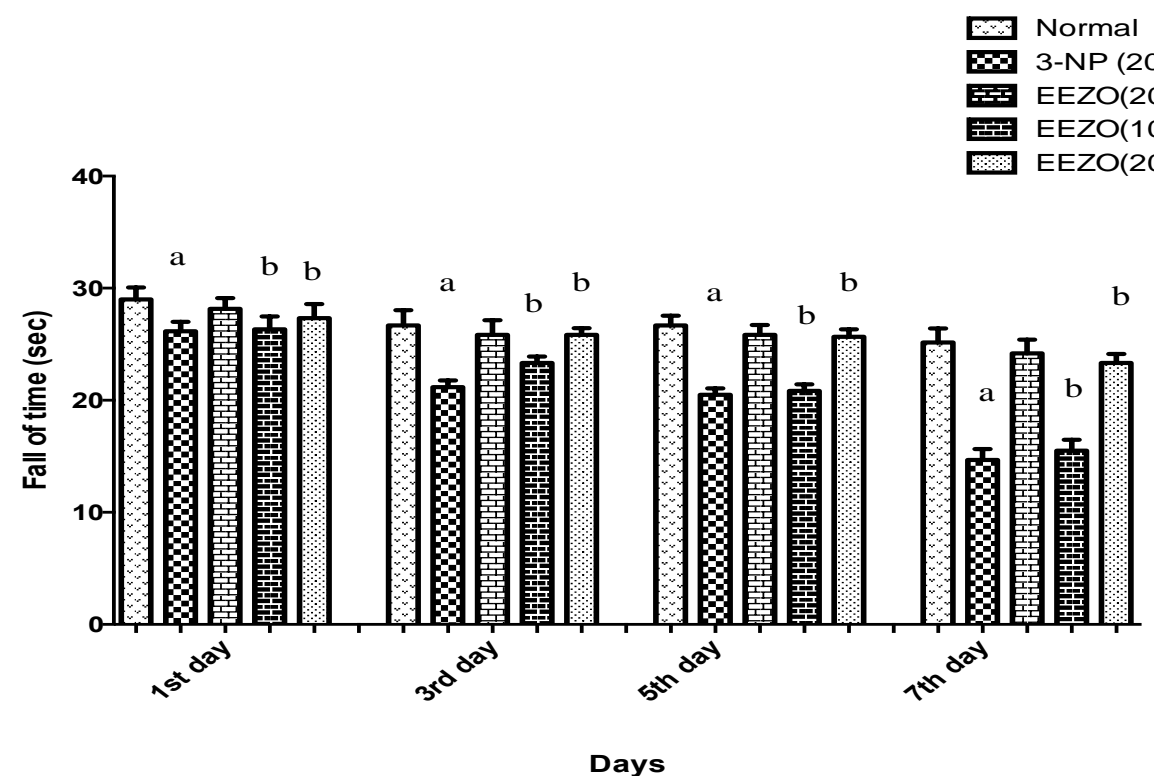

Fig 2 Effect of EEZO (100 and $200 \mathrm{mg} / \mathrm{kg}$ ) on Rotarod activity in 3-NP administered rats. Data are mean \pm SEM values ( $n=6$ in each group). $a=p<0.05$ compared to vehicle-treated normal group; $b=p<0.05$ compared to 3-NP-injected group (one-way ANOVA followed by Tukey test) 


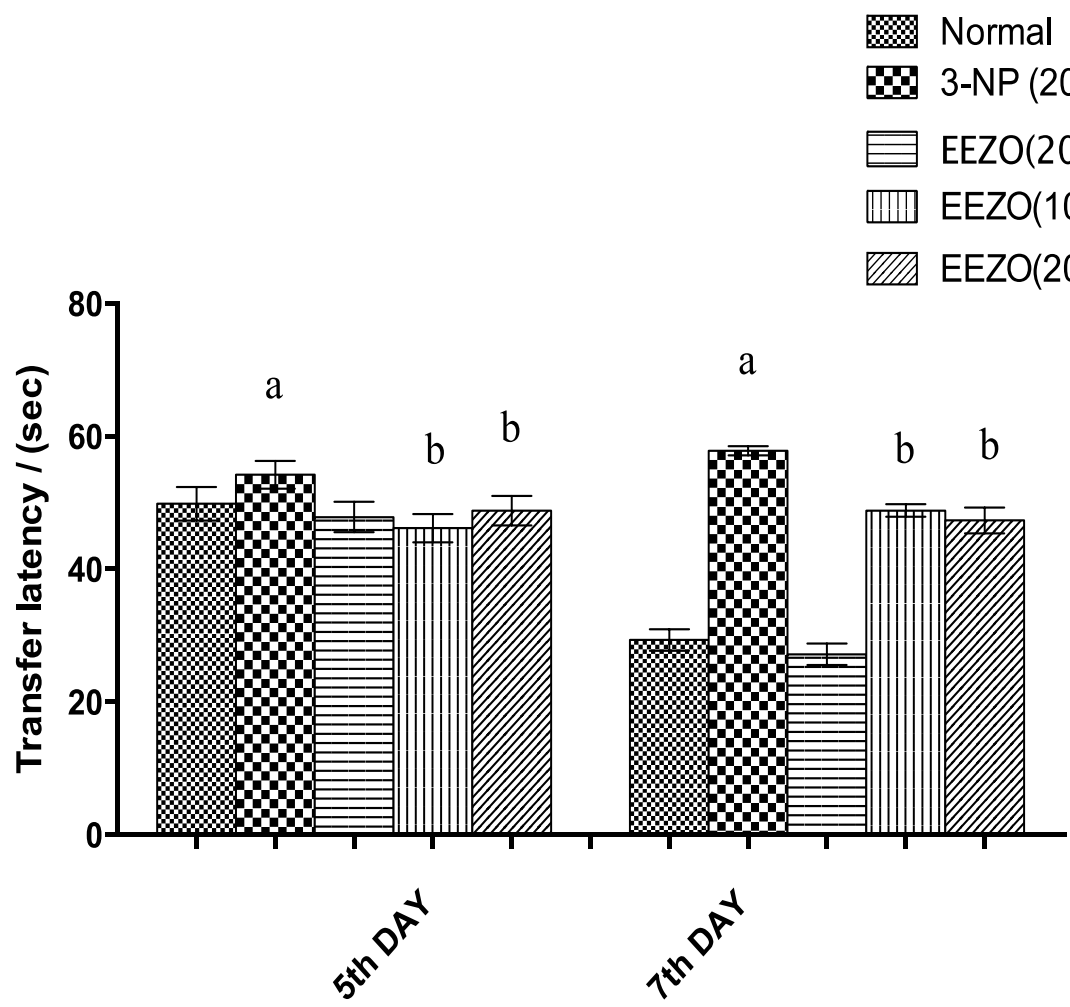

Fig 3 Effect of EEZO (100 and $200 \mathrm{mg} / \mathrm{kg}$ ) on elevated plus maze activity in 3-NP administered rats. Data are mean \pm SEM values ( $\mathrm{n}=6$ in each group). $\mathrm{a}=\mathrm{p}<0.05$ compared to vehicle-treated normal group; $b=p<0.05$ compared to 3-NP injected group (one-way ANOVA followed by Tukey test)
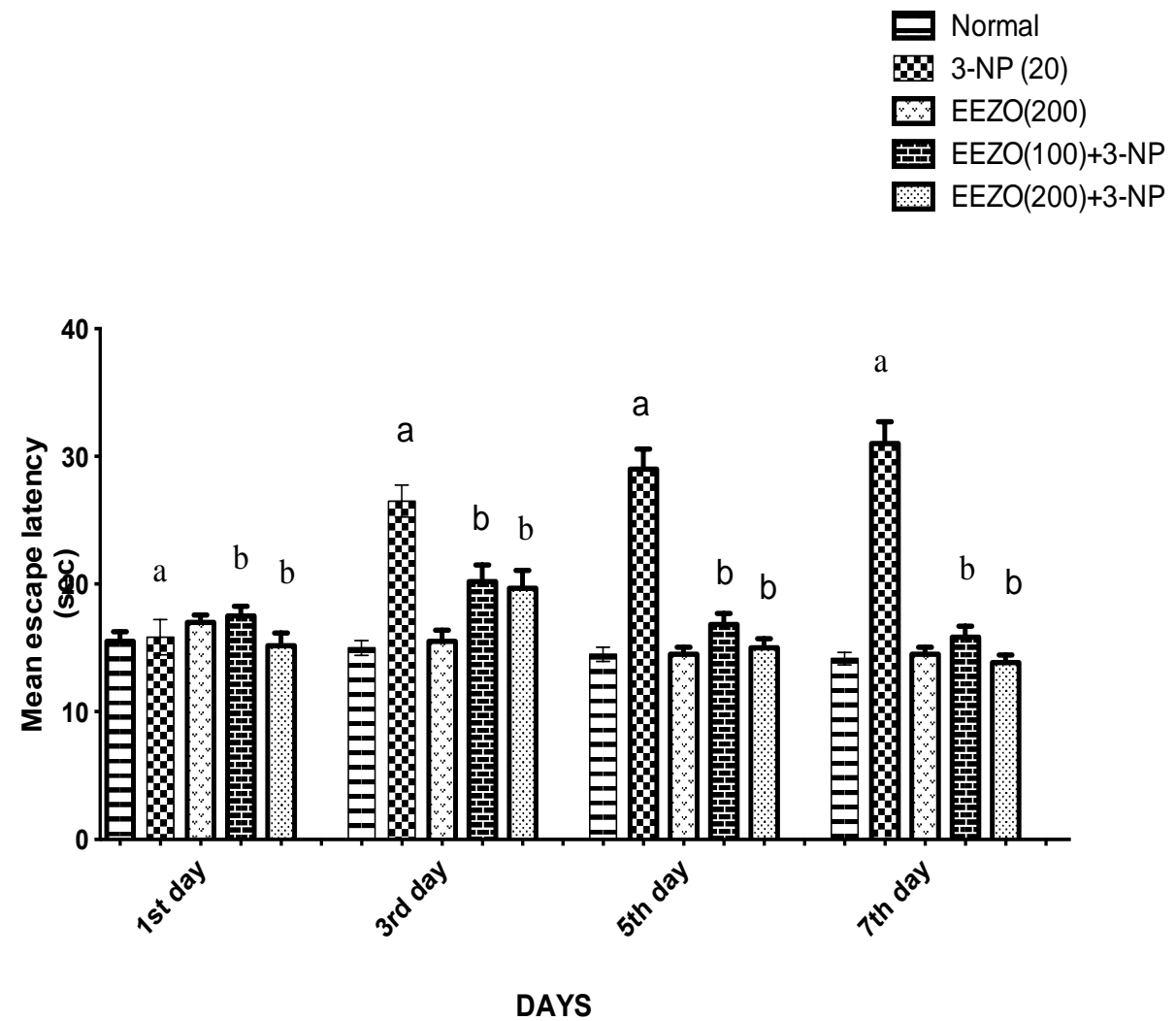

Fig 4 The effect of the administration of EEZO on time spent by rat to reach submerged quadrant or( we check for )transfer latency in MWM. All values are represented as mean \pm S.E.M. $a=p<0.001 \mathrm{v} / \mathrm{s} \mathrm{Normal}$; $b=p<0.001$ (one-way ANOVA followed by Tukey test) 


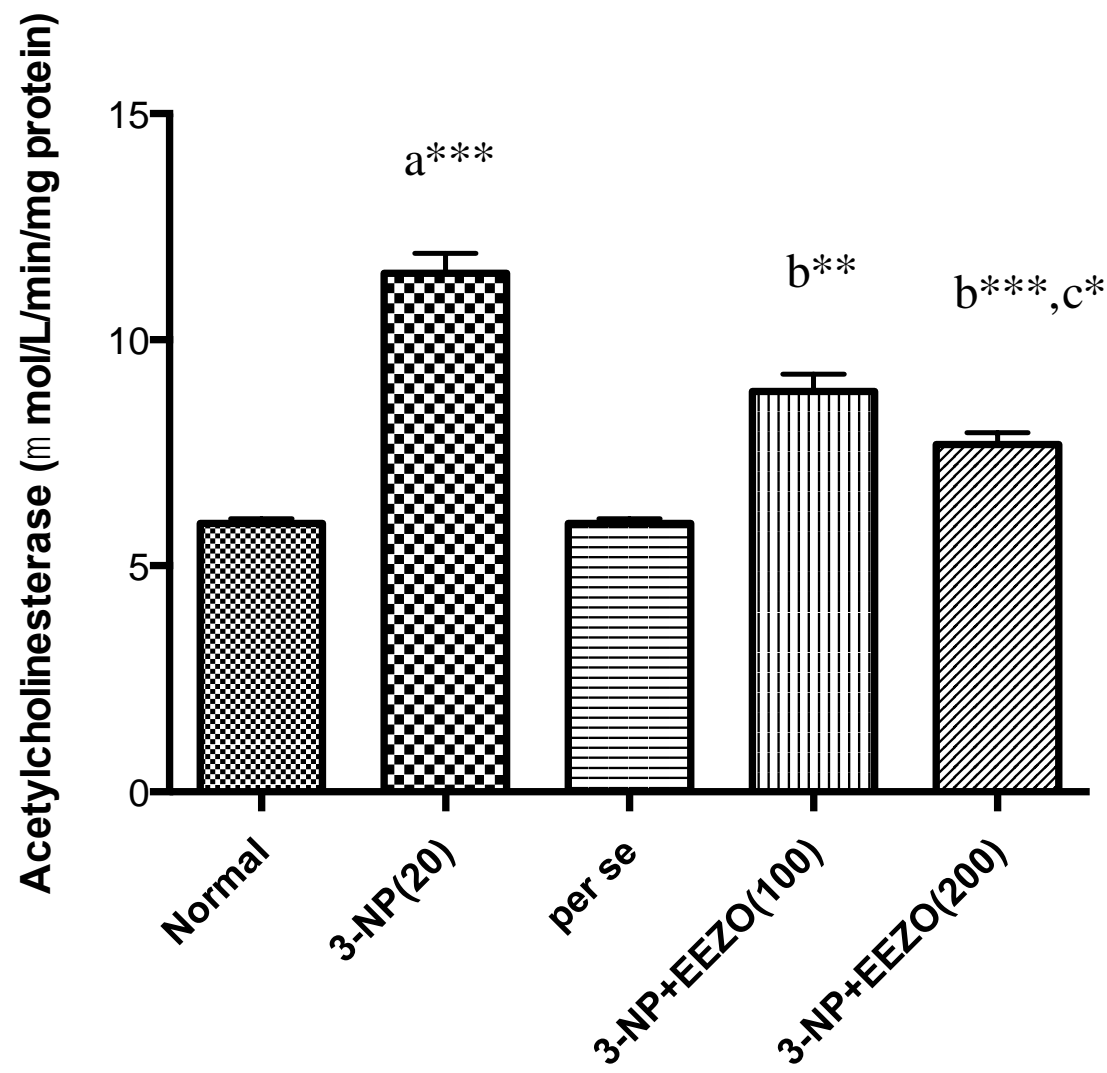

Fig 5 The effect of administered EEZO on AChE level. Data are mean \pm SEM values ( $n=6$ in each group). $a=p<0.05$ compared to vehicle-treated normal group; $b=p<0.05$ compared to 3-NP injected group; $c=p<$ 0.05 compared to (3-NP+100 mg/kg) group (one-way ANOVA followed by Tukey test)

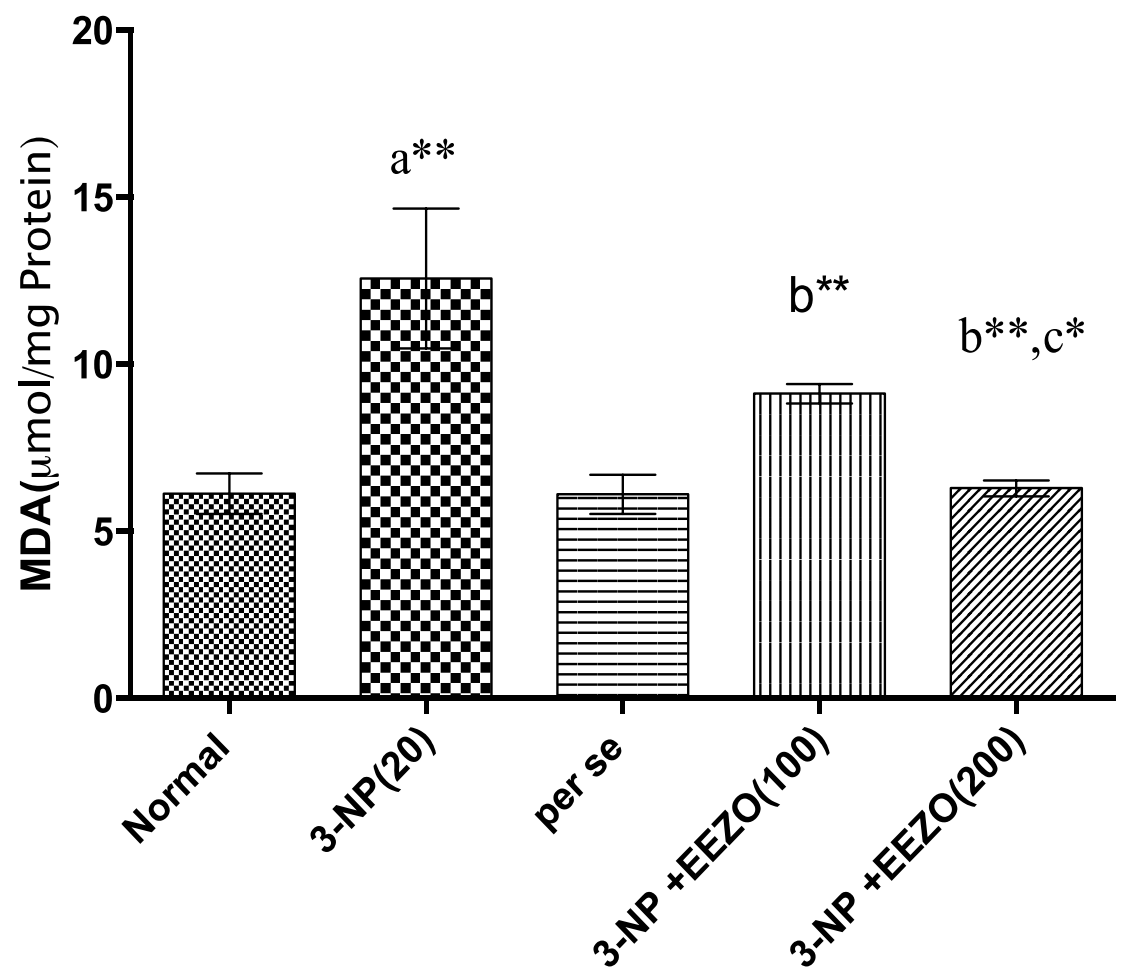

Fig 6 The effect of administered EEZO on Lipid Peroxide level. Data are mean \pm SEM values $(n=6$ in each group). $a=p<0.05$ compared to vehicle-treated normal group; $b=p<0.05$ compared to $3-\mathrm{NP}$ injected group; $\mathrm{c}=\mathrm{p}<0.05$ compared to $(3-\mathrm{NP}+100 \mathrm{mg} / \mathrm{kg})$ group (one-way ANOVA followed by Tukey test) 


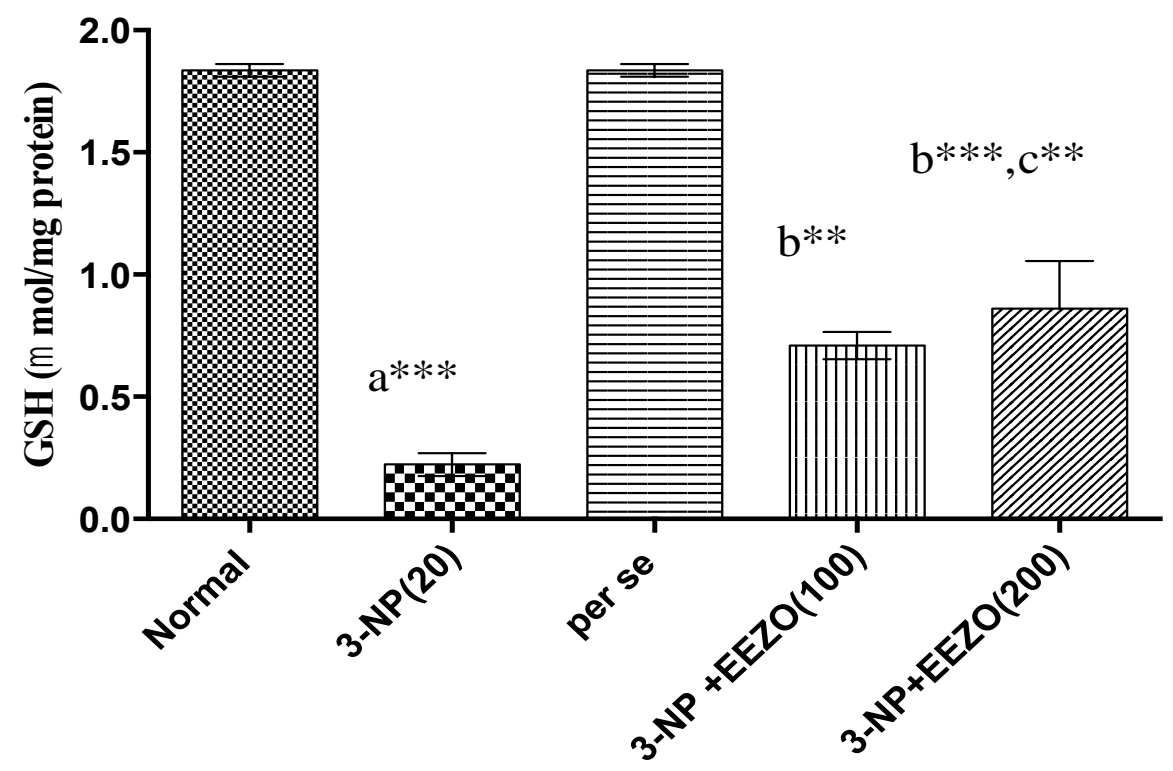

Fig 7 The effect of administered EEZO on GSH level. Data are mean \pm SEM values ( $n=6$ in each group). $a=p<0.05$ compared to vehicle-treated normal group; $b=p<0.05$ compared to 3-NP injected group; $c=p<$ 0.05 compared to (3-NP+100 mg/kg) group (one-way ANOVA followed by Tukey test)

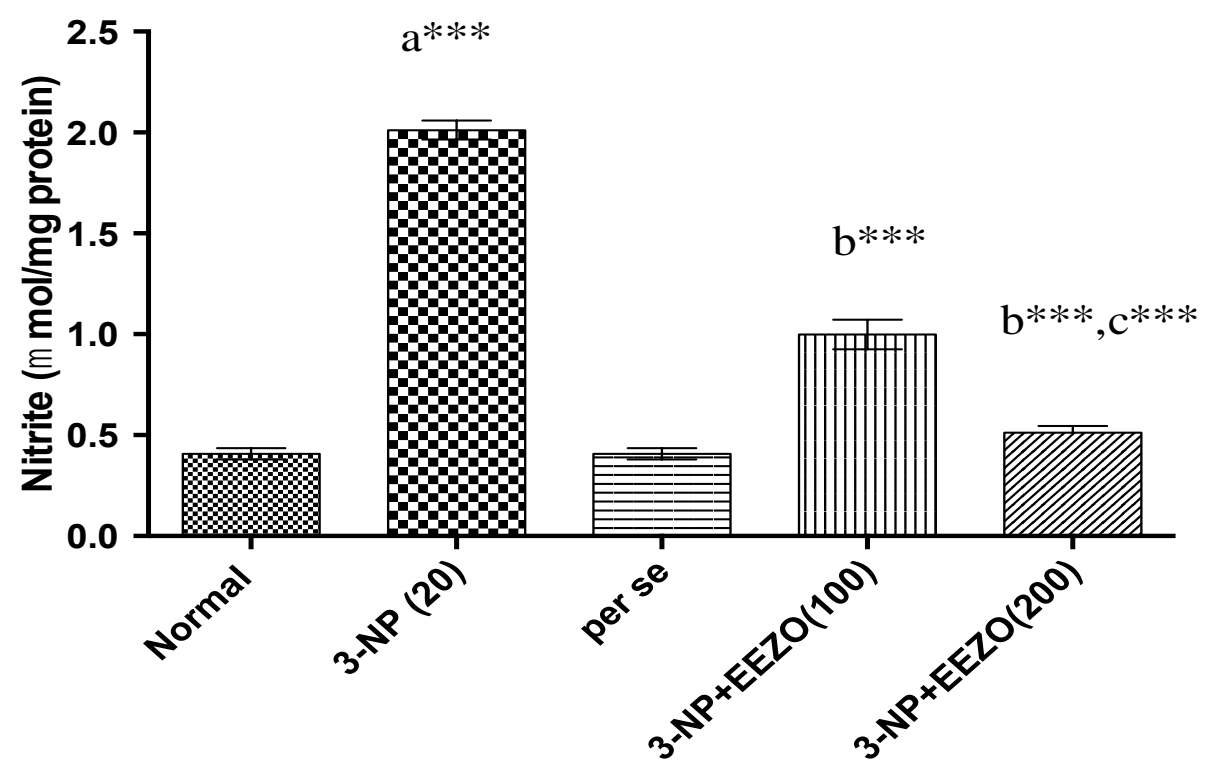

Fig 8 The effect of administered EEZO on Nitrite level. Data are mean \pm SEM values ( $n=6$ in each group). $a=p<0.05$ compared to vehicle-treated normal group; $b=p<0.05$ compared to 3-NP injected group; $c=p<$ 0.05 compared to $(3-\mathrm{NP}+100 \mathrm{mg} / \mathrm{kg}$ ) group (one-way ANOVA followed by Tukey test) 


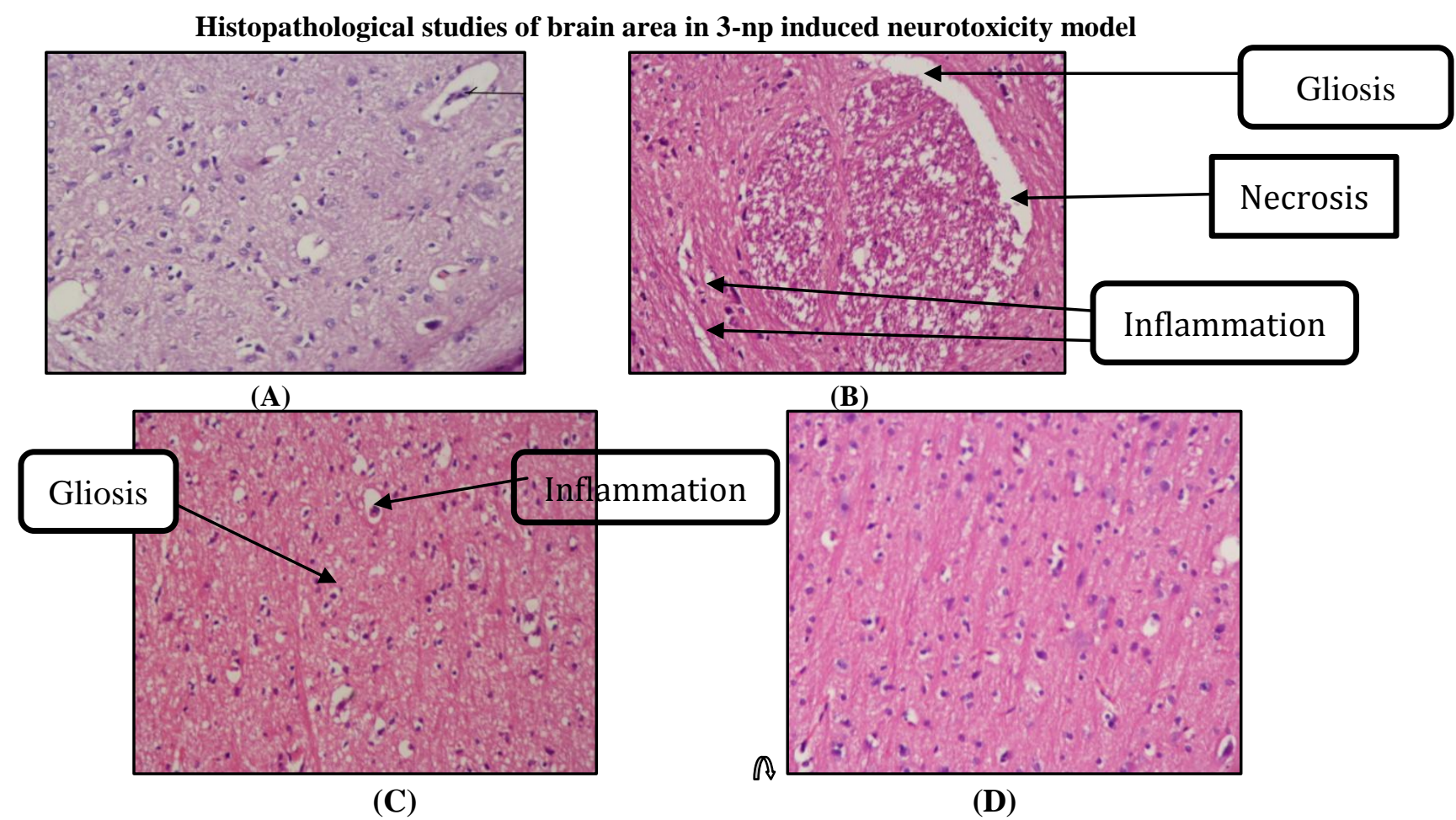

Fig. 9.. Effect of EEZO on 3-nitropropionic acid induced histopathological changes in rat brain striatum. Histological sections of rat striatum stained with $\mathrm{H} \& \mathrm{E}$ : (A) - Normal groupwith no pathological changes (B) 3-NP(20mg/kg), showed gliosis, inflammation and Necrosis (C) EEZO(100mg/kg)+3-NP,shows mild changes (D) EEZO $(200 \mathrm{mg} / \mathrm{kg})+3$-NP, showed no pathological changes .

\section{DISSCUSSION}

$\mathrm{HD}$ is an autosomal dominant inherited disorder characterized by progressive decline in motor and cognitive functions (35). HD is characterized by destruction of cholinergic receptors leading to decreased level of acetylcholine (36). The exact pathogenesis of the disease is not yet known, but evidence suggest that impairment in energy metabolism and oxidative stress play a major role in the pathogenesis of the Huntington's disease (37). However, the events which prompt or intervene the loss of Ach neurons remain unclear. Plants pose an important and a safer alternative for the management of neurodegenerative disorders (,38). Even, World Health Organization (WHO) has also documented the importance of folk medicine and has created strategies, guidelines and standards for botanical medicines. Thus, the present study examined the therapeutic value of antioxidants in the prevention of 3-NP neurotoxicity. Our results demonstrated that 3-NP administration increases the oxidative stress through lipid peroxidation and subsequently decreasing level of endogenous antioxidants. Ginger, a potent antioxidant, can be protective in this model of neurotoxicity depicted by significantly improved cognitive defects, prevented oxidative stress induced by systemic 3 -NP treatment.

3-NP is a neurotoxin that irreversibly inhibits succinate dehydrogenase (SDH), a relevant enzyme constituting the complex II of the respiratory chain during mitochondrial electron transport (39). 3-NP is known to produce oxidative/nitrosative stress and evokes an experimental model of Huntington's disease $(40,41,42,43)$. Indeed, a growing body of evidence suggests oxidative stress as a culprit in mediating behavioral impairment and memory deficits in age-related neurodegenerative disorders (44). This is too supported in our study that $3 \mathrm{NP}$ administered rats showed a significant decrease in transfer latency with subsequent impairment in motor coordination and muscle grip strength. Further, 3-NP treated rats there is marked increase in the level of TBARS, marker of lipid peroxidation, nitric oxide (NO) with attenuation in the level of reduced glutathione. This is supported by earlier reports that 3-NP administration generated oxidative/ nitrosative stress (46). Further, it is well reported that 3-NP produces lesions in hippocampal CA1 and CA3 pyramidal neurons, the area of brain that is associated with cognitive performance $(45,46,47)$ which is too revealed in our study by performing histopathology of brain. 3-NP administration to rats results in increased activity of acetylcholinesterase enzyme that reduces the level of Ach in the stratitum, that is the basic reason of HD (48).

Our results demonstrated that treatment with Z.officinale a neuroprotectant(49), dose-dependently enhances the memory with improvement in the locomotor and muscle grip strength in 3-NP-administered rats. 
Accumulating evidences indicates that ginger showed marked protection in various neurological disease models (50).

Recently, it has been reported that ginger prevents oxidative stress and improves impaired cognitive performance in animals This is too supported in our study that treatment with Zingiber officinale increased the level of GSH and attenuated TBARS with NO, marker of oxidative and nitrosative stress. Further, Zinger officinale treatment had a significantly $(\mathrm{p}<0.001)$ increased the level of acetylcholine by inhibiting AChE.

Thus, it can be concluded that being a very potent antioxidant, Z.officinale too possess anticholinestersae potency, that is responsible for improving memory orcognitive functions. Hence, the neuroprotective role of Zingiber officinale may be due to its anticholinesterase activity.

\section{CONCLUSION}

Zingiber officinale was found to possess a therapeutic effect against Huntington's disease in 3NPinduced animal HD models. Thus being a very potent antioxidant zinger too possesses anticholinestersae potency, that is responsible for improving memory orcognitive functions. Further studies with different extracts and their fractions are encoured to identify the chemical constituents responsibe for nueroprotection in Huntington's disease.

\section{ACKNOWLEDGEMENT}

The authors would like to thank, The Director, Rayat Institute of Pharmacy (Punjab) for providing the necessary facilities for the research work .

\section{REFERENCES}

[1]. Huntington G. On chorea. Med Surg Rep Philadelphia 1872, pp: 317-21.

[2]. Ferrante RJ. Mouse model of huntington's disease and methodological considerations for therapeutic trials. Biochimica et BiophysicaActa 2009;1792:506-20.

[3]. Gil, J.M., Rego, A.C., 2008. Mechanisms of neurodegeneration in Huntington's disease.Eur. J. Neurosci. 27, 28032820.

[4]. Hoth, K.F., Paulsen, J.S., Moser, D.J., Tranel, D., Clark, L.A., Bechara, A., 2007. Patients with Huntington's disease have impaired awareness of cognitive, emotional and functional abilities. J. Clin. Exp. Neuropsychol. 29, 365-376.ropsychologia 44, 1984-1994.

[5]. Montoya, A., Price, B.H., Menear, M., Lepage, M., 2006b. Brain imaging and cognitive dysfunctions in Huntington's disease. J. Psychiatr. Neurosci. 31, 21-29.

[6]. Cooper, D.B., Ales, G., Lange, C., Clement, P., 2006. Atypical onset of symptoms in Huntington disease: severe cognitive decline preceding chorea or other motor manifestations. Cogn. Behav. Neurol. 19, 222-224.

[7]. Montoya, A., Pelletier, M., Menear, M., Duplessis, E., Richer, F., Lepage, M., 2006a. Episodic memory impairment in Huntington's disease: a meta-analysis. Neu- ropsychologia 44, 1984-1994.

[8]. Cruz V and Santamaria A. Integrative Hypothesis for Huntington's disease: A Brief Review of Experimental Evidence. Physiol Res 2007;56:513-26.

[9]. Beal, M.F., Brouillet, E., Jenkins, B.G., Ferrante, R.J., Kowall, N.W., Miller, J.M., Storey, E., Srivastava, R., Rosen, B.R., Hyman, B.T., 1993. J. Neurosci. 13, 4181-4192.

[10]. Kumar, P., Padi, S.S., Naidu, P.S., Kumar, A., 2007. Possible neuroprotective mechan-isms of Curcumin in attenuating 3-nitropropionic acid-induced neurotoxicity.Methods Finding Exp. Clin. Pharmacol. 29, 1-7.

[11]. Browne SE, Ferrante RJ, Beal MF. Oxidative stress in Huntington's disease.Brain Pathol 1999;9:147-63.

[12]. Silva-Adaya D, Pérez-De La Cruz V, Herrera-Mundo MN, Mendoza-Macedo K, Villeda- Hernández J, Binienda $\mathrm{Z}$, et al. Excitotoxic damage, disrupted energy metabolism, and oxidative stress in the rat brain: antioxidant and neuroprotective effects Neurotoxicol. Teratol. 17, 333-339. of L- carnitine. J Neurochem 2008;105(3):677-89.

[13]. Fu, Y., He, F., Zhang, S., Huang, J., Zhang, J., Jiao, X., 1995. 3-Nitropropionic acid produces indirect excitotoxic damage to rat striatum.

[14]. Zeevalk, G.D., Derr-Yellin, E., Nicklas, W.J., 1995. Relative vulnerability of dopamine and GABA neurons in mesencephalic culture to inhibition of succinate dehydrogenase by malonate and 3-nitropropionic acid and protection by NMDA receptor blockade.J. Pharmacol. Exp. Ther. 275, 1124-1130.

[15]. Lee WT, Chang C. Magnetic resonance imaging and spectroscopy in assessing 3- nitropropionic acid-induced brain lesions: an animal model of Huntington's disease. ProgNeurobiol 2004;72:87-110

[16]. Kumar, P., Padi, S.S., Naidu, P.S., Kumar, A., 2007. Possible neuroprotective mechanisms of Curcuminin attenuating 3-nitropropionic acid-induced neurotoxicity. Methods Finding Exp. Clin.Pharmacol. 29, 1-7.

[17]. Raso GM, Meli R, Di Carlo G, Pacilio M, Di Carlo R. Inhibition of inducible nitric oxide synthase and cyclooxygenase-2 expression by flavonoids in macrophage J774A.1. Life Sci 2001;68:921-31

[18]. Awang, D.V.C., 1992. Ginger. Can. Pharm. J. 125, 309-311.

[19]. Wang, W.H., Wang, Z.M., 2005. Studies of commonly used traditional medicine-ginger. ZhongguoZhong Yao ZaZhi. 30, 1569-1573.

[20]. Chrubasik, S., Pittler, M.H., Roufogalis, B.D., 2005.Zingiberisrhizoma: a comprehensive review on the ginger effect and efficacy profiles. Phytomedicine 12, 684-701. 
[21]. Tapsell, L.C., Hemphill, I., Cobiac, L., Patch, C.S., Sullivan, D.R., Fenech, M., Roodenrys, S., Keogh, J.B., Clifton, P.M., Williams, P.G., Fazio, V.A., Inge, K.E., 2006.

[22]. Health benefits of herbs and spices: the past, the present, the future. Med. J. Aust. 185 (Suppl. 4), S4-S24.

[23]. Hanan M. Abd El Gawad, Hanan S. El-Abhar2 Nadia M. S. Arafa .Cysteamine in 3- nitropropionic acid model of Huntington's disease in rats: Modulation of mitochondrial function and amino acid pattern .International Journal of Medicine and Medical Sciences June 2009 Vol 1(6) pp. 254-262,.

[24]. Afzal, M., Al-Hadidi, D., Menon, M., Pesek, J., Dhami, M.S., 2001. Ginger: an ethnomedical, chemical and pharmacological review. Drug Metab. Drug Interact. 18, 159-190.

[25]. Kim, J.K., Kim, Y., Na, K.M., Surh, Y.J., Kim, T.Y., 2007. [6]-Gingerol prevents UVB-induced ROS production and COX-2 expression in vitro and in vivo. Free Radic. Res. 41, 603-614.

[26]. Kumar P and Kumar A. Protective effect of rivastigmine against 3-NP induced HD like symptoms. European J Pharmacol 2009;615:91-101.

[27]. Kumar P and Kumar A. Protective effect of rivastigmine against 3-NP induced HD like symptoms. European J Pharmacol 2009;615:91-101.

[28]. Reddy, D.S., Kulkarni, S.K., 1998. Possible role of nitric oxide in the nootropic and antiamnesic effects of neurosteroids on aging and dizociline induced leaning impairment. Brain Res. 799, 215-219.

[29]. Neuroprotective effect of cyclosporine and FK506 against 3-nitropropionic acid induced cognitive dysfunction and glutathione redox in rat: Possible role of nitric oxide Puneet Kumar and Anil Kumar .Neuroscience Research 63 (2009) 302-314

[30]. Hiroshi ohkawa, Nobuko ohishi, and Kunioyagi .Assay for Lipid Peroxides in Animal Tissues by Thiobarbituric Acid Reaction .Analytical Biochemistry 95.351-358(1979)

[31]. Ellman, G.L., Courtney, K.D., Andres, V., Featherstone, R.M., 1961. A new and rapid colorimetricdetermination of acetylcholinesterase activity.Biochem. Pharmacol. 7, 88-9.

[32]. Beal, M.F., Brouillet, E., Jenkins, B.G., Ferrante, R.J., Kowall, N.W., Miller, J.M., Storey, E., Srivastava, R., Rosen, B.R., Hyman, B.T., 1993. J. Neurosci. 13, 4181-4192.

[33]. Kumar P, Padi SSV, Naidu, PS and Kumar A. Effect of resveratrol on 3-nitropropionic acid- induced biochemical and behavioural changes: possible neuroprotective mechanisms. BehavPharmacol 2006;17:485-92

[34]. Kumar P, Padi SSV, Naidu, PS and Kumar A. Effect of resveratrol on 3-nitropropionic acid- induced biochemical and behavioural changes: possible neuroprotective mechanisms. BehavPharmacol 2006;17:485-92.

[35]. S. Nakamura, T. Takahashi, H. Yamashita, H. Kawakami, Nicotinic acetylcholine receptors and neurodegenerative disease, Alcohol 24 (2001) 79-81.

[36]. KumarP,KaloniaH,KumarA.Sesamolattenuate3-nitropropionicacid-induced Huntington-like behavioral, biochemical, and cellular alterations in rats. J Asian Nat Prod Res 2009;11:439-50.

[37]. Browne SE, Ferrante RJ, Beal MF. Oxidative stress in Huntington's disease.Brain Pathol 1999;9:147-63.

[38]. Beal, M.F., Brouillet, E., Jenkins, B.G., Ferrante, R.J., Kowall, N.W., Miller, J.M., Storey, E., Srivastava, R., Rosen, B.R., Hyman, B.T., 1993. J. Neurosci. 13, 4181-4192.

[39]. Kumar P, Padi SSV, Naidu, PS and Kumar A. Effect of resveratrol on 3-nitropropionic acid- induced biochemical and behavioural changes: possible neuroprotective mechanisms. BehavPharmacol 2006;17:485-92.

[40]. Kumar P, Padi SSV, Naidu PS and Kumar A. Possible Neuroprotective of Curcumin in Attenuating 3Nitropropionic Acid -Induced Neurotoxicity. Methods Find Exp Clin. Pharmacol 2007a;29:1-7.

[41]. Tunez I, Feijoo M, Collado JA, Medina FJ, Pena J, Munoz MC, Jimena I, Franco F, Rueda I, Muntane J and Montilla P. Effect of testosterone on oxidative stress and cell damage induced by 3-nitropropionic acid in striatum of ovariectomized rats. Life Sciences 2007

[42]. Tunez I, Collado JA, Medina FJ, Peña J, Del C Muñoz M, Jimena I, Franco F, Rueda I, Feijóo M, Muntané J, Montilla P. $17 \beta$-Estradiol may affect vulnerability of striatum in a 3-nitropropionic acid-induced experimental model of Huntington's disease in ovariectomized rats. Neurochem Intern 2006b;48: 367-73.

[43]. Mohammad Tariq, Haseeb Ahmad Khan, Ibrahim Elfaki, Saleh Al Deeb, Khalaf Al Moutaery Brain Research Bulletin 67 (2005) 161-168 Neuroprotective effect of nicotine against 3-nitropropionic acid (3-NP)-induced experimental Huntington's disease in rats 2007;80:1221-7.

[44]. Miller PJ, Zaborszky L. 3-Nitropropionic acid neurotoxicity: visualization by silver staining and implications for use as an animal model of Huntington's disease. Exp Neurol 1997;146:212-29.

[45]. Duan W, Guo Z, Mattson MP. Participation of par-4 in the degeneration of striatal neurons induced by metabolic compromise with 3-nitropropionic acid. Exp Neurol 2000;165:1-11. )

[46]. Kumar P and Kumar A. Protective effect of rivastigmine against 3-NP induced HD like symptoms. European J Pharmacol 2009;615:91-101.

[47]. Abeer $m$ waggas .Nueroprotectiveevalautaion of extract of (zingiber offcinale) pakisthan $\mathrm{j}$ of biological sciences 12(3):201 212,2009.root in monosodium glutamate toxicity in different areas in male albino rats .

[48]. ReinhardGrzanna, PhongPhan, Anna Polotsky, Lars Lindmark, and Carmelita G. Frondoza. Ginger Extract Inhibits $\beta$-Amyloid Peptide-Induced Cytokine and Chemokine Expression in Cultured THP-1 Monocytes .The Journal of Alternative and Complementary Medicine. December 2004, 10(6) 